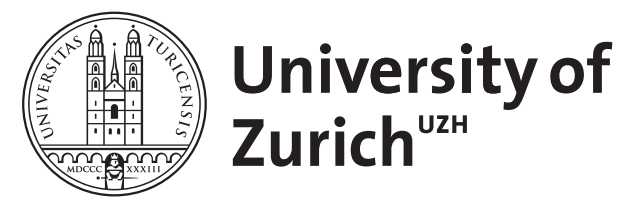

\title{
The time course of processing external and internal features of unfamiliar faces
}

\author{
Veres-Injac, Bozana ; Schwaninger, Adrian
}

\begin{abstract}
The time course of processing internal and external facial features was studied in a sequential face matching task, where first a target face was presented, followed by a test face. The exposure duration of the test face was varied systematically $(90,120,150 \mathrm{~ms}$, and self-paced). In three tasks, participants were instructed to match either the whole face, only external features, or only internal features of the target and test face. Taken together, the results in all the three tasks provide evidence for very fast matching processes. For upright faces, maximal performance was achieved at 90ms exposure duration and longer exposure durations $(120,150 \mathrm{~ms}$, self-paced) did not improve accuracy. For inverted whole faces, reduced exposure duration resulted in an increase of matching errors, suggesting that below $150 \mathrm{~ms}$ of exposure duration, inverted faces cannot be matched reliably. When matching selected facial features only, no such inversion effect was found. Our data challenges previous claims that external features are matched faster than internal: no difference of time course was found between external and internal features. However, external features were matched more accurately
\end{abstract}

DOI: https://doi.org/10.1007/s00426-008-0147-5

Posted at the Zurich Open Repository and Archive, University of Zurich

ZORA URL: https://doi.org/10.5167/uzh-155675

Journal Article

Published Version

Originally published at:

Veres-Injac, Bozana; Schwaninger, Adrian (2009). The time course of processing external and internal features of unfamiliar faces. Psychological Research, 73(1):43-53.

DOI: https://doi.org/10.1007/s00426-008-0147-5 


\title{
The time course of processing external and internal features of unfamiliar faces
}

\author{
Bozana Veres-Injac $\cdot$ Adrian Schwaninger
}

Received: 22 August 2007 / Accepted: 25 March 2008 / Published online: 18 April 2008

(C) Springer-Verlag 2008

\begin{abstract}
The time course of processing internal and external facial features was studied in a sequential face matching task, where first a target face was presented, followed by a test face. The exposure duration of the test face was varied systematically $(90,120,150 \mathrm{~ms}$, and selfpaced). In three tasks, participants were instructed to match either the whole face, only external features, or only internal features of the target and test face. Taken together, the results in all the three tasks provide evidence for very fast matching processes. For upright faces, maximal performance was achieved at $90 \mathrm{~ms}$ exposure duration and longer exposure durations $(120,150 \mathrm{~ms}$, self-paced) did not improve accuracy. For inverted whole faces, reduced exposure duration resulted in an increase of matching errors, suggesting that below $150 \mathrm{~ms}$ of exposure duration, inverted faces cannot be matched reliably. When matching selected facial features only, no such inversion effect was found. Our data challenges previous claims that external features are matched faster than internal: no difference of time course was found between external and internal features. However, external features were matched more accurately.
\end{abstract}

B. Veres-Injac $(\varangle) \cdot$ A. Schwaninger

Department of Psychology, University of Zurich,

Binzmühlestrasse 14/22, 8050 Zurich, Switzerland

e-mail: b.veres@psychologie.uzh.ch

A. Schwaninger

School of Applied Psychology (APS),

Institute Humans in Complex Systems (MikS),

University of Applied Sciences, Northwestern Switzerland

(FHNW), Riggenbachstrasse 16, 4600 Olten, Switzerland

\section{Introduction}

Humans are supposed to recognize faces with astonishing ease and speed (Seeck et al. 1997). However, less is known about the time scale and operations occurring in the early stages of face processing. Neuropsychological studies have shown that visual ERP (event related potential) for facial stimuli is elicited within less than 200 ms (Jeffreys 1989; Eger, Jedynak, Iwaki, \& Skrandies 2003; Keysers, Xiao, Foldiak, \& Perrett 2001). The time course of face processing has also been the subject of behavioral studies. Yin (1970) found a disproportionate reduction of recognition performance under brief presentation $(100 \mathrm{~ms})$ which is similar to the disproportionate effect of inversion in faces. Hole (1994) used vertical and horizontal chimeric faces, which were presented for 80 or $2,000 \mathrm{~ms}$. He suggested that long exposure duration allows feature by feature comparison, while short exposure duration forces participants to use configural strategies to process the face as a whole (Hole 1994).

In our study, we used behavioral methods to investigate the sequence of processes involved in matching unfamiliar faces. Our focus lay in the processing of external and internal features in the whole facial context. External facial features refer to the hair, ears, head- and face-outline, whereas internal features refer to the eyes, eyebrows, nose and mouth. Three hypotheses were tested:

(1) The first hypothesis deals with the effect of orientation. The claims regarding the inversion effect for isolated internal or external features are ambiguous. It has been argued that since inversion impairs configural (spatial relationship between features) but not featural processing (e.g. hair, face outline, eyes, mouth, nose etc.), recognition of facial features in inverted faces should not be impaired (for a review see Valentine 1988; Schwaninger, Carbon, \& Leder 2003). However, the inversion effect was found for isolated 
facial features (Barton, Keenan \& Bass 2001; Rakover \& Teucher 1997) as well as for the external and internal features (Nachson \& Shechory 2002; Moscovitch \& Moscovitch 2000). In all of these studies the external and internal features were presented isolated, without the whole facial context. Consequently, the conclusions gained from those studies cannot be applied to the processing of the external and internal features within the whole facial context. The contribution of the facial features to face perception can be studied only in the context of whole faces, where not only featural but also configural or holistical information is available (Nachson, Moscovitch, \& Umilta 1995). According to Nachson et al. (1995), upright faces are processed on the basis of their configurations in the sense that various features interact to produce an integrated facial stimulus. They, however, did not investigate if there is any configural integration of the facial features if stimuli are presented inverted. Moreover, black and white line drawings were used as stimuli, which are not a realistic representation of a face. The aim of our study was to investigate whether orientation impairs matching of internal or external features when they are shown in a facial context of a photographic image. If the configural relations are weaker or completely lost in inverted orientation we may expect no effect of inversion for matching of only internal or only external facial features.

We were also interested in how exposure duration affects mathcing of upright and inverted faces. There are two reasons why such an effect could be expected: first, there is evidence that reduction of ERP response for identical inverted faces is smaller and delayed by $30 \mathrm{~ms}$ in comparison with identical upright faces (Jacques, d'Arripe, \& Rossion 2007). Second, if inverted faces are processed by a serial part-by-part strategy, which is also time-consuming, we might expect an interaction between exposure duration and inversion. Barton et al. (2001) have found that inversion effect was reduced with prolonged exposure duration, whereas the performance for upright faces stayed unaffected. However, they used rather long exposure durations (1, 2, $3 \mathrm{~s}$ and self-paced) which do not have direct implication for early face processing (Barton et al. 2001).

(2) An interaction between feature configuration and the exposure duration was addressed in this study too. Matching performance is expected to be strongly impaired by the features of the faces being compared. A match in only internal or external features of two faces is found to be more difficult than the matching of wholly identical or wholly different faces (Nachson et al. 1995). The matching of faces sharing only external or internal features ("same external", SE; "same internal", SI) requires more part-bypart comparisons, whereas two faces, which are either identical (ID) or have no common features at all ("different", DF) could be processed more holistically. This seems to be even more so since the reaction times are longer for the condition SE and SI than for ID and DF (Nachson et al. 1995). Consequently, the matching of two faces sharing only external (SE) or internal (SI) features is expected to be more impaired by short exposure duration than matching of identical or different faces.

(3) The studies of the microgenesis of perception led to the hypothesis that global aspects of objects are processed faster than their details (Love, Rouder, \& Wisniewski 1999; Hubner 1997; Hoeger 1997). In face recognition, most of the studies have revealed faster and more accurate matching of external than internal features (De Haan \& Hay 1986; Nachson et al. 1995; Young, Hay, McWeeny, Flude, \& Ellis 1985). Internal features contain featural (eyes, eyebrows, nose, mouth) as well as configural information (distances between those features), while external features contain more global information. According to this assumption, internal features are expected be more impaired by short exposure duration than external features, because the former contain more information and have a more detailed structure. External features refer to the global aspects of the face and therefore are expected to be less impaired by short exposure duration.

To test our hypotheses, we measured behavioral performance in three different matching tasks: matching of whole faces (Task 1) and matching only external (Task 2) or only internal (Task 3) features. A sequential matching task was used, with constant exposure duration for the target faces $(1,500 \mathrm{~ms})$, and four different exposure durations for the test faces (90, 120, $150 \mathrm{~ms}$ and self-paced). The short exposure durations were varied within the range of the ERP time course for faces (Jeffreys 1989; Eger et al. 2003; Keysers et al. 2001). Presentation of the target and test faces were separated by $1,000 \mathrm{~ms}$ of an interstimulus interval (ISI) for two reasons. First, we aimed to avoid a participant's use of traces in iconic memory, which facilitates complete pictorial matching instead of face matching. It has been showed that representation in iconic memory lasts up to 200 $300 \mathrm{~ms}$ and decays after that (Schilller 1968; Rolls \& Tovee 1994; Kovacs, Vogels \& Orban 1995; Keysers, Xiao, Foldiak, \& Perret 2005; Martens, Schweinberger, Kiefer, \& Burton 2006). After $1,000 \mathrm{~ms}$ no traces of the first presented, target face is expected to be present in iconic memory. Secondly, self-paced exposure duration, where the second face was exposed until the response, was performed to compare performance between short and long exposure durations. A mask cannot be used in this test condition. To keep test conditions comparable, we did not use a mask for the short exposure durations (90, 120, $150 \mathrm{~ms})$. In spite of lacking the mask we believe that the exposure duration should still have an effect on the general performance. As it is well established, information present in iconic memory is pre-categorical and if to be processed an item must be represented in post-categorical form (for a review 
see Coltheart 1983). Moreover, the transfer to post-categorical stage depends strongly on the number of items and on the capacity limits of working memory (Coltheart 1977, 1983; Fuster \& Jervey 1981). Our tasks are rather complex, requiring comparisons on a part-to-whole basis relying strongly on the memory for both target and test faces. That is why we expect that viewing exposure durations in combination with the long ISI between the two faces should affect matching performance, even if the visual persistence was not controlled by a mask. To ensure that the pictorial matching and the lack of the mask did not influence general performance, we conducted a control experiment where the whole identity of the two faces was matched within the $150 \mathrm{~ms}$ exposure durations. In the control experiment the second presented picture of the face was $17 \%$ smaller than the first and a mask was presented for $100 \mathrm{~ms}$ directly after the second face. Matching of the whole identity between faces is supposed to be easier than the part-based matching of isolated features (Nachson et al. 1995). Therefore, if the lack of a mask does not affect matching of the whole face in Task 1, then we can argue that it would have an even smaller influence on the other two part-based matching tasks (matching of external or internal features).

\section{Method}

\section{Participants}

A total of 38 students (age 20-35 years), participated in the three experiments introduced above: 10 in Task 1 (9 females), 14 in Task 2 (10 females), and 14 in Task 3 (11 females). In the control experiment, 10 students ( 7 females) were all unaware of the purpose of the experiment and none had participated in the previous three experiments. All the participants had normal or corrected to normal vision.

\section{Stimuli}

Full-color frontal view photographs of 12 male faces were captured in a photo studio under controlled lighting conditions using the same background. None of the faces was wearing glasses and jewelry or had a beard. The haircut for all faces was kept comparably similar with short hairstyle. Four faces were chosen as the target faces and were paired with the appropriate 12 test faces. For each of the 4 target faces there were 12 test faces respectively. Three of the test faces were identical with the target face, three were sharing the same internal features as the target faces, three were with the same external features and three of the test faces were completely different from the test face. A total of possible pairs were 48 , the test faces were assigned to four conditions. In the identical (ID) condition, the same face was used as test and target face. In the same external (SE) condition, both target and test faces shared the same external features, while internal features differed. In the same internal (SI) condition, only the internal features of the target and test faces were the same. In the different (DF) condition, target and test faces depicted two different persons with no common features at all. Figure 1 shows an example of a target and three corresponding test faces.

The test faces for condition SE and SI were prepared using Adobe Photoshop 9. The internal features were cut out with comparable tracing lines and placed on the second (template) face, based on the position of the internal features. All the stimuli were $14.11-\mathrm{cm}$ long and $10.58-\mathrm{cm}$ wide with the resolution 72 pixels/in. Images were presented on a color $17^{\prime \prime}$ CRT monitor. Screen resolution was set to $1,024 \times 768$ pixels with the refresh rate of $60 \mathrm{~Hz}$. The viewing distance was approximately $60 \mathrm{~cm}$.

\section{Procedure}

The participants were tested individually in the experimental room. All the participants first completed a short practice to become familiar with the task. All were tested in two sessions, once with upright and once with inverted stimuli (both target and test faces), and were randomly assigned to one of the two procedures (upright first versus inverted first). Between two sessions, there was a break of 7 days to avoid a learning effect. One session took about $30 \mathrm{~min}$ and contained 192 trails. Each trial started with a target face that was displayed for $1,500 \mathrm{~ms}$, and after a blank ISI of $1,000 \mathrm{~ms}$, a test

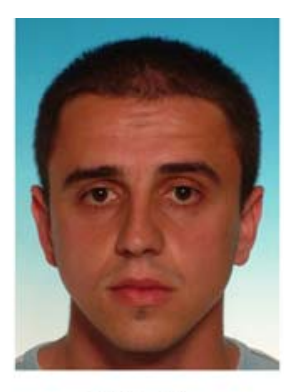

Target

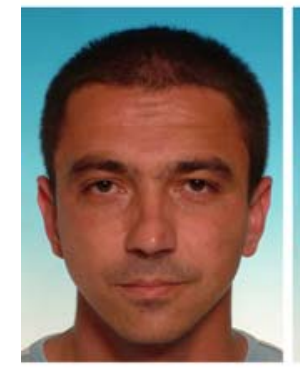

Same External

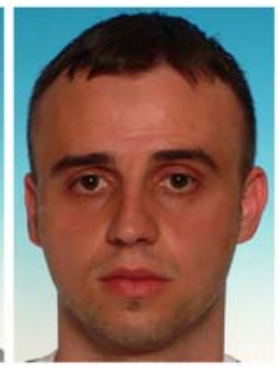

Same Internal

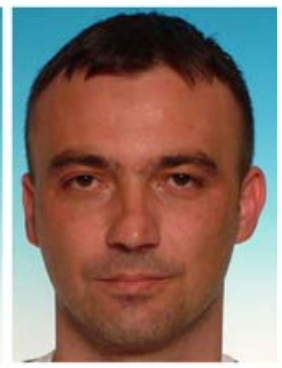

Different
Fig. 1 Sample of a target face and three test faces 
face appeared. Images were presented centered using SuperLab 2.0. Participants were asked to respond as quickly and accurately as possible whether the target and the test faces were the same or different by pressing the left or right mouse button with their preferred hand. The assignment of answers (same/different) to the left or right mouse button was counterbalanced across participants. In Task 1, same was defined as whole congruency between target and test faces, including both internal and external features. In Task 2, same was defined as congruency of external features only, and in Task 3 as congruency of internal features only. Participants completed either Task 1, or 2, or 3. Each test face was shown four times, once for $90,120,150 \mathrm{~ms}$, and once until the participants' answer occur (self-paced condition). Four blocks with different exposure durations were randomized across participants, while condition (ID, SI, SE, DF) was randomized across trials. In the control experiment matching of whole faces was required (as in the Task 1), whereas the exposure duration was constant at $150 \mathrm{~ms}$ and the test face was $17 \%$ smaller than the target face.

\section{Results}

The reaction times of correct responses and the number of errors were recorded for each trial. The data of the three tasks were combined and subjected to a 4-factor analysis of variance (ANOVA) with orientation (upright vs. inverted), exposure durations $(90,120,150 \mathrm{~ms}$ and self-paced) and conditions (ID, SE, SI, DF) as within-participants factors and task (same $=$ wholly identical, same $=$ identical internal features only, same $=$ identical external features only) as between-participant factor. Bonferroni correction was conducted for multiple comparisons. Additional analyses were performed where necessary as reported below.

\section{Average error}

Results for repeated ANOVA for the mean matching errors are shown in the Table 1.

The analysis of matching errors revealed significant differences between the three tasks, whereas pairwise comparisons showed that participants were less accurate in Task 3 (matching of internal features) than in Task 1 (matching of whole faces) and Task 2 (matching of external features) (both $p<0.001)$. There was no significant difference in matching errors between Task 1 and Task 2. Average matching errors for all the three tasks are depicted in Fig. 2.

The main effect of condition was also significant as well as an interaction between task and condition. For identicaltrails (ID), the average matching error was significantly higher than for matching of SE-, SI- or DF-trails in Task 1
Table 1 Repeated ANOVA for the mean matching errors

The Task (matching of whole faces, external or internal facial features) was a between-participants variable and orientation, exposure duration and conditions were within-participants variables. Significant main effects and interactions are assigned

\begin{tabular}{|c|c|c|c|c|c|}
\hline & SS & $d f$ & MS & $F$ & $p$ \\
\hline (1) Task & 1.85027 & 2 & 0.92513 & 15.2445 & 0.000017 \\
\hline Error & 2.12402 & 35 & 0.06069 & & \\
\hline (2) Orientation & 0.47761 & 1 & 0.47761 & 7.1732 & 0.011194 \\
\hline Orientation $\times$ task & 0.31861 & 2 & 0.15931 & 2.3926 & 0.106181 \\
\hline Error & 2.33036 & 35 & 0.06658 & & \\
\hline (3) Exposure duration & 0.15054 & 3 & 0.05018 & 5.4118 & 0.001679 \\
\hline Exposure duration $\times$ task & 0.02774 & 6 & 0.00462 & 0.4986 & 0.808174 \\
\hline Error & 0.97357 & 105 & 0.00927 & & \\
\hline (4) Condition & 2.31503 & 3 & 0.77168 & 20.0912 & 0.000000 \\
\hline Condition $\times$ task & 5.81416 & 6 & 0.96903 & 25.2294 & 0.000000 \\
\hline Error & 4.03291 & 105 & 0.03841 & & \\
\hline Orientation $\times$ exposure duration & 0.10931 & 3 & 0.03644 & 2.8212 & 0.042472 \\
\hline Orientation $\times$ exposure duration $\times$ task & 0.05278 & 6 & 0.00880 & 0.6811 & 0.665221 \\
\hline Error & 1.35616 & 105 & 0.01292 & & \\
\hline Orientation $\times$ condition & 0.33050 & 3 & 0.11017 & 3.5904 & 0.016165 \\
\hline Orientation $\times$ condition $\times$ task & 0.23306 & 6 & 0.03884 & 1.2659 & 0.279444 \\
\hline Error & 3.22174 & 105 & 0.03068 & & \\
\hline Exposure duration $\times$ condition & 0.13954 & 9 & 0.01550 & 1.4266 & 0.175520 \\
\hline Exposure duration $\times$ condition $\times$ task & 0.19895 & 18 & 0.01105 & 1.0169 & 0.439925 \\
\hline Error & 3.42359 & 315 & 0.01087 & & \\
\hline Orientation $\times$ exposure duration $\times$ condition & 0.08150 & 9 & 0.00906 & 0.9319 & 0.497398 \\
\hline $2 \times 3 \times 4 \times 1$ & 0.17507 & 18 & 0.00973 & 1.0009 & 0.458233 \\
\hline Error & 3.06088 & 315 & 0.00972 & & \\
\hline
\end{tabular}


Fig. 2 Average matching errors for: a Task 1 -matching of whole faces, b Task 2-matching of external features and $\mathbf{c}$ Task 3-matching of internal features in upright and inverted orientation. Average number of errors are plotted against the four exposure durations and broken up by conditions (ID, SE, SI, $\mathrm{DF})$
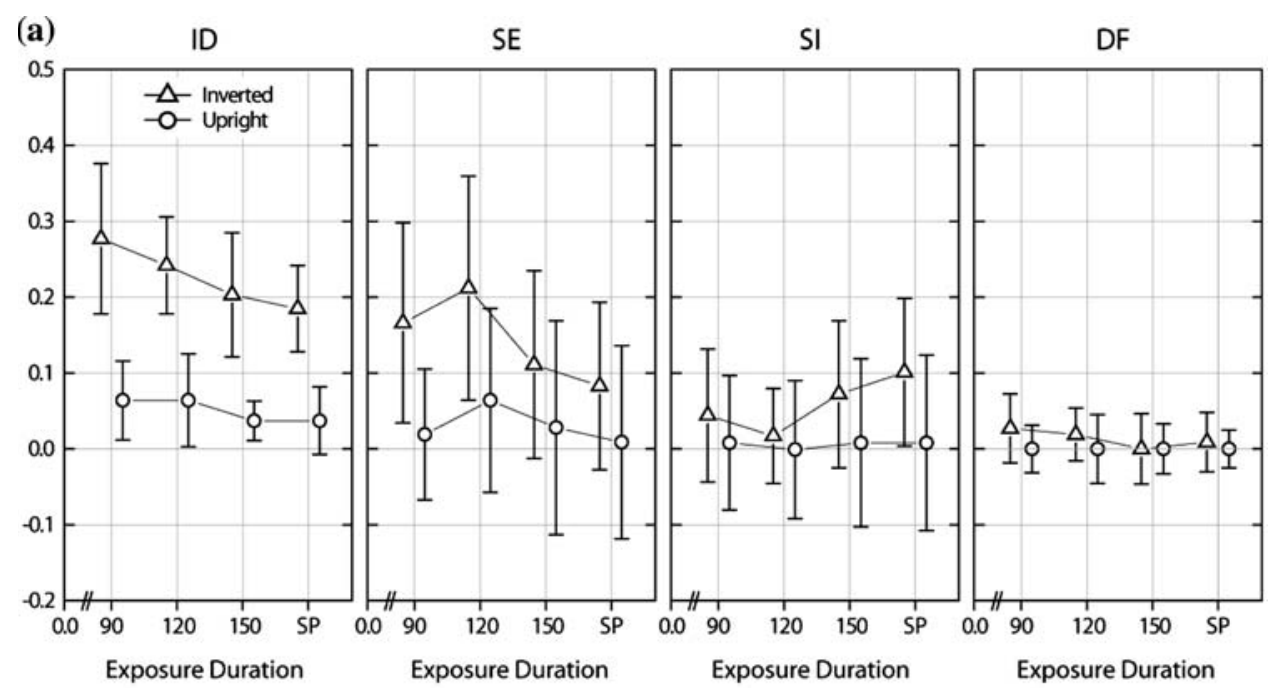

(b)

ID

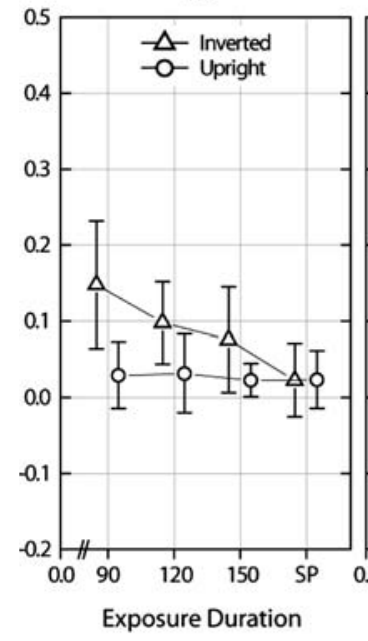

SE

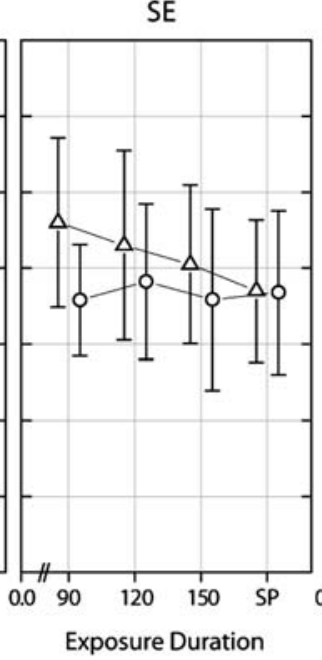

SI

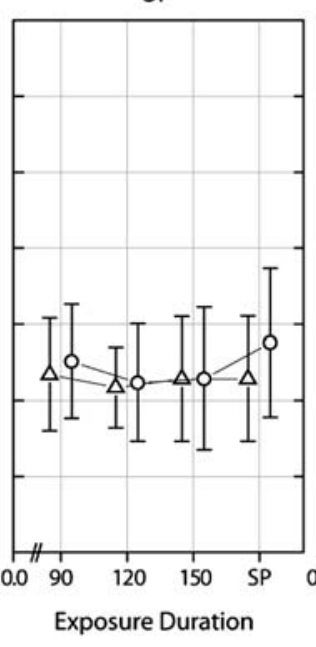

DF

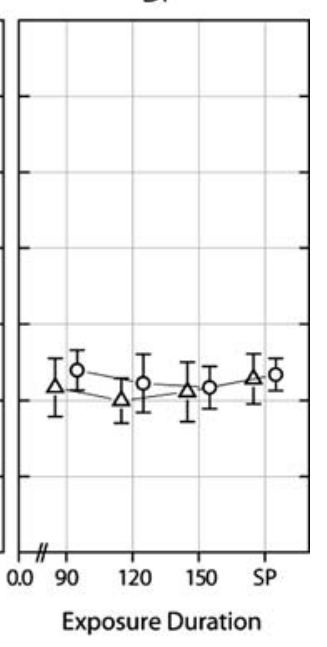

(c)

ID

SE

SI
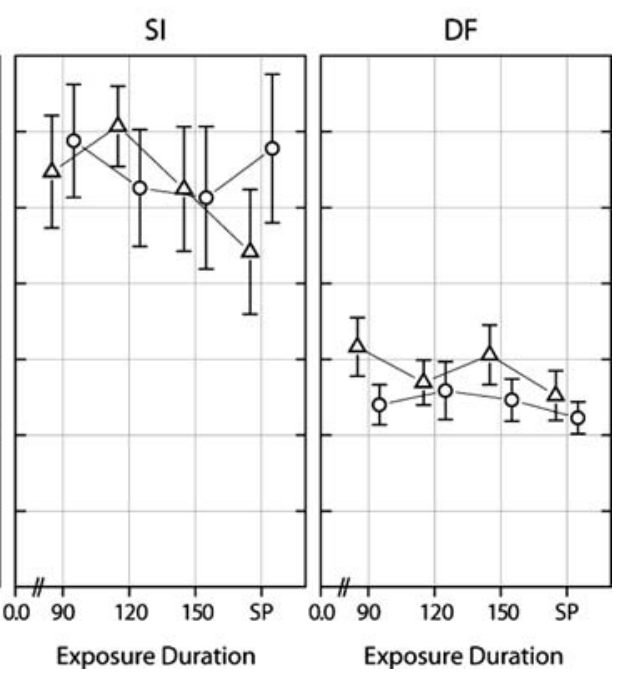

(same $=$ identical faces). In the Task 2 (same $=$ same external features) matching of the SE-trails was related with significantly more matching errors than conditions ID, SI and
DF. In Task 3 (same = same internal features) the highest matching error was found for SI-trails in comparison with the other three conditions (all $p<0.001$ ). 
Overall, the performance for upright faces was more accurate than for inverted ones and there was a significant interaction between orientation and condition. The simple effect of orientation was significant only for ID-trials $F(1$, $37)=21.39 ; p<0.001$, whereas there were no orientation effect for SE-trials $(F(1,37)=1.64, p=0.201)$, SI-trials $(F(1,37)=0 ; p=0.98)$, and DF-trials $(F(1,37)=2.4$; $p=0.1 .2)$. A separate analysis for each of the tasks showed that the inversion effect reached statistical significance only in Task 1: $F(1,9)=23.11, p<0.001$, where whole faces had to be matched. In Task 2 (matching of external features) and Task 3 (matching of internal features), there was no significant inversion effect [Task 2: $F(1,13)=1.20$, $p=0.29$; Task 3: $F(1,13)=.13, p=0.72]$.

The main effect of exposure duration was significant in the sense that matching errors decreased with the increased exposure durations. The pairwise comparisons revealed a significant difference in matching errors between $90 \mathrm{~ms}$ and self-paced condition. Moreover, there was a significant interaction between orientation and exposure duration. The simple effect of exposure duration was found for inverted but not for upright stimuli: $F(3,105)=7.48, p<0.001$. Pairwise comparisons showed that there was no significant difference $(p=0.88)$ in matching errors between self-paced in comparison with the other three exposure durations (90, 120 and $150 \mathrm{~ms}$ ) if stimuli are presented upright. The same pairwise comparisons for inverted presented stimuli showed a significant effect of exposure durations $(p<0.001)$, with a significant difference appearing below $150 \mathrm{~ms}$ exposure durations.

\section{Reaction times}

The results of the repeated ANOVA are shown in the Table 2, whereas the mean reactions times are depicted in the Fig. 3.

Analysis of variance revealed no differences in reaction times between the three tasks suggesting that whole faces as well as internal and external facial features were processed equally fast. Overall, reaction times were faster for upright than for inverted presentations. Although an interaction between orientation and condition for reaction times approximated statistical significance, the analysis of simple effects showed the same pattern as found for accuracy: the inversion effect for ID-trials was significant, $F(1,37)=8.7$, $p<0.001$, while for SE-, SI- and DF-trials, there was no significant effect of orientation. Separate analyses for each of the three tasks revealed significant effects of orientation only in Task 1 (matching of whole faces): $F(1,9)=23.11$, $p<0.001$. There was no orientation effect in Task 2 (matching of external features): $F(1,13)=.12, p<0.74$, and Task 3 (matching of internal features), $F(1,13)=1.15, p<0.30$.
Table 2 Repeated ANOVA for mean matching RTs was conducted

The same between- and withinparticipants variables were used as in the ANOVA for error rate. Significant differences are assigned

\begin{tabular}{|c|c|c|c|c|c|}
\hline & SS & $d f$ & MS & $F$ & $p$ \\
\hline (1) Task & 187,903 & 2 & 93,951 & 0.0923 & 0.912052 \\
\hline Error & $35,625,717$ & 35 & $1,017,878$ & & \\
\hline (2) Orientation & 334,6384 & 1 & 334,6384 & 4.3003 & 0.045528 \\
\hline Orientation $\times$ task & 318,6297 & 2 & $1,593,148$ & 2.0473 & 0.144263 \\
\hline Error & $27,236,224$ & 35 & 778,178 & & \\
\hline (3) Exposure duration & 724,2625 & 3 & $2,414,208$ & 17.4781 & 0.000000 \\
\hline Exposure duration $\times$ task & 877,752 & 6 & 146,292 & 1.0591 & 0.391928 \\
\hline Error & $14,503,406$ & 105 & 138,128 & & \\
\hline (4) Condition & 4630,302 & 3 & $1,543,434$ & 17.3648 & 0.000000 \\
\hline Condition $\times$ task & $10,579,306$ & 6 & $1,763,218$ & 19.8376 & 0.000000 \\
\hline Error & $9,332,681$ & 105 & 88,883 & & \\
\hline Orientation $\times$ exposure duration & 56,747 & 3 & 18,916 & 0.1831 & 0.907701 \\
\hline Orientation $\times$ exposure duration $\times$ task & 611,375 & 6 & 101,896 & 0.9861 & 0.438553 \\
\hline Error & $10,849,868$ & 105 & 103,332 & & \\
\hline Orientation $\times$ condition & 610,201 & 3 & 203,400 & 2.5240 & 0.061656 \\
\hline Orientation $\times$ condition $\times$ task & 582,862 & 6 & 97,144 & 1.2054 & 0.309347 \\
\hline Error & 8461,731 & 105 & 80,588 & & \\
\hline Exposure duration $\times$ condition & 2255,958 & 9 & 250,662 & 6.8455 & 0.000000 \\
\hline Exposure duration $\times$ condition $\times$ task & 1500,543 & 18 & 83,364 & 2.2766 & 0.002450 \\
\hline Error & $11,534,450$ & 315 & 36,617 & & \\
\hline Orientation $\times$ Exposure duration $\times$ condition & 175,066 & 9 & 19,452 & 0.7349 & 0.676851 \\
\hline $2 \times 3 \times 4 \times 1$ & 602,885 & 18 & 33,494 & 1.2654 & 0.208768 \\
\hline Error & 8337,975 & 315 & 26,470 & & \\
\hline
\end{tabular}


Fig. 3 Average RTs for: a Task 1 -matching of whole faces, $\mathbf{b}$ Task 2-matching of external features and $\mathbf{c}$ Task 3 -matching of internal features in upright and inverted orientation. RTs in $\mathrm{ms}$ are plotted against the four exposure durations $(90,120$, $150 \mathrm{~ms}$, self-paced) and broken up by conditions
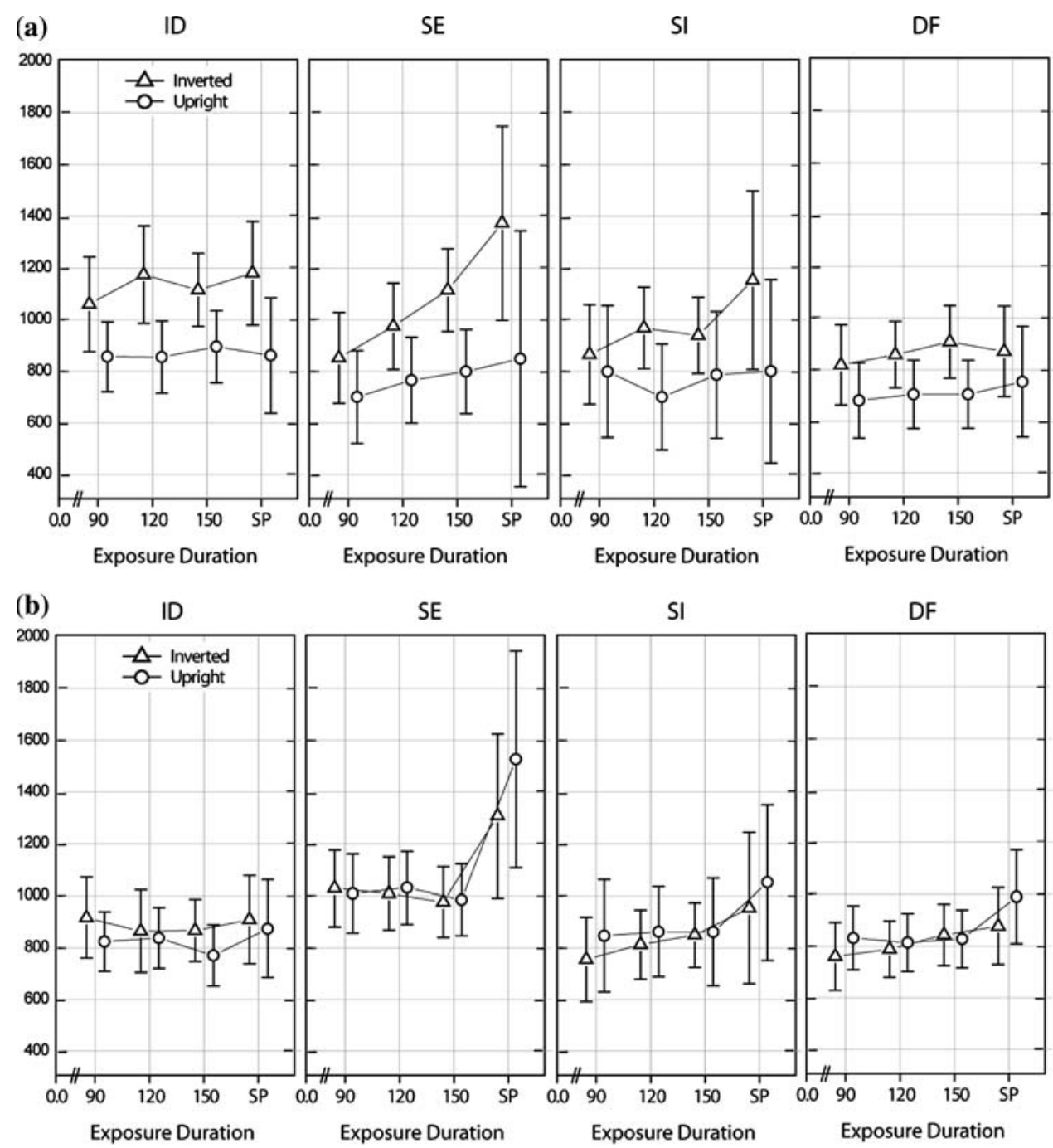

(c)
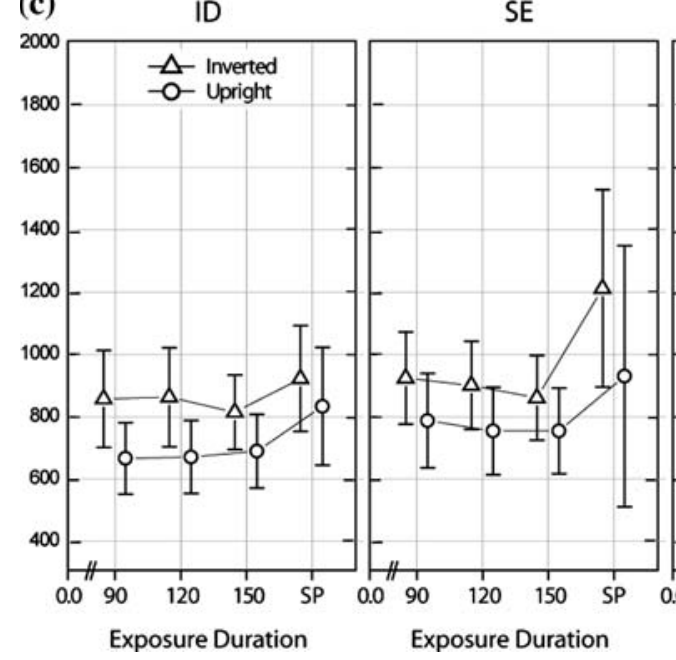

SI

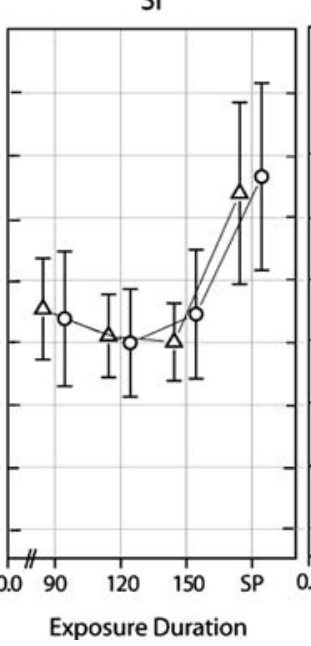

DF

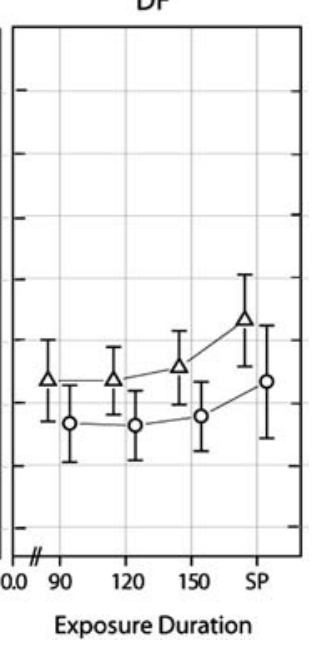

The main effect of condition and an interaction between task and condition were significant. As for accuracy pairwise comparisons showed that the reaction time was the longest for ID-trials in the Task 1 (matching of the whole faces), SE-trials in Task 2 (matching of external features) and SI-trials in Task 3 (matching of the internal features, all $p<0.001$ ).

The main effect of exposure duration was also significant with the significantly longer reaction time for self-paced 
exposure duration than for 90,120 and $150 \mathrm{~ms}$. However, there was a significant two-way interaction between exposure duration and condition as well as a three-way interaction between task, exposure duration and condition. To analyze the three-way interaction, we conducted separate ANOVAs for each of the three tasks: in Task 1 (matching of whole faces), the increase of exposure duration results in longer reaction times, but only for SE-trials $[F(3$, $27)=7.18, p<0.001]$ and SI-trials $[F(3,27)=3.08$, $p<0.05$ ], while ID and DF-trials were not influenced by exposure duration (both $p>1.64$ ). In Task 2 (matching of external features) the increase of exposure duration results in longer reaction times in the SE, SI and DF-trials [SE: $F(3,39)=4.43, p<0.01$; SI: $F(3,39)=3.79, p<0.05$; DF: $F(3,39)=3.67, p<0.05]$, but not in ID-trials $(p=0.60)$. In Task 3 (matching of internal features), all the four conditions (ID, SE, SI, DF) were impaired by exposure duration (all $p<0.05$ ).

Trade-off between reaction times and matching errors

To test a possible speed-accuracy trade-off, correlations between RTs and matching errors were calculated. There were some significant correlations, but all of them positive, showing that speed-accuracy trade-offs did not occur in our data.

\section{Control experiment}

A control experiment was conducted to test the effect of a mask and compare how the lack of a mask influences matching performance in the previous three experiments. Due to long ISI interval and the high cognitive load in our matching task we did not expect any significant impact. In the control experiment the exposure duration was constant at $150 \mathrm{~ms}$, while the test face was resized to be $17 \%$ smaller than the target face. After the test face was presented a pattern mask was shown for $100 \mathrm{~ms}$. Mean matching errors and mean reaction times from the control experiment were compared with the data for $150 \mathrm{~ms}$ exposure duration in Task 1, where whole faces had to be matched. Repeated ANOVA was conducted on mean matching errors and mean reaction times, with experiment (Task 1 vs. control experiment) as between-participants variable and the orientation (upright vs. inverted) and condition (ID; SE; SI; DF) as within-participants variables. For error rate there was no significant difference in matching errors between the two experiments $[F(1,18)=0.51 ; p=0.48]$, whereas the reaction time was significantly faster in the control experiment where the mask was presented $[F(1,18)=24.71$; $p<0.001]$. We can therefore conclude that the error rate in the control experiment is not caused by longer reaction time. A different computer was used to conduct the control experiment, which may also contribute to the faster reaction time. Nevertheless, the main effects and global trends are the same in both data sets and lead to the conclusion that the lack of the mask did not cause a significant difference in the general performance. Mean matching errors and mean reaction times for both experiments are shown in Fig. 4.

\section{Discussion}

In this study, we investigated the effects of inversion and short exposure duration on the processing of internal and external facial features in the whole facial context. The tasks explored matching of two faces on the basis of their internal features only, of their external features only, or on the basis of both internal and external features (whole face matching).

As the first hypothesis we have tested how orientation impairs matching of whole faces and their facial features. To draw a conclusion about the contribution of the external and internal facial features to the processing of the upright and inverted faces, it seems reasonable to explore it in the context of the whole faces, as we have done it in this study. According to the holistic or configural approach, the inversion effect is expected only for whole faces, but not for facial features (for a review see Valentine 1988; Schwaninger et al. 2003). However, there are studies revealing an inversion effect when isolated facial features were presented (Rakover \& Teucher 1997; Moscovitch \& Moscovitch 2000; Barton et al. 2001; Nachson \& Shechory 2002). The data we obtained showed a higher average error rate and longer reaction time for the processing of inverted than for upright faces. Further analysis revealed an inversion effect only for whole face matching (Task 1), while matching of external or internal features remained unimpaired (Task 2 and Task 3). So far, our findings support the hypothesis that inversion impairs holistic or configural processing, while featural processing remains unaffected. Thereby our data seems to contradict earlier studies, which imply the same cognitive processes for inverted whole faces as well as for their internal and external features (Rakover \& Teucher 1997; Moscovitch \& Moscovitch 2000; Nachson \& Shechory 2002).

At this point it is important to notice that in our stimuli, the facial proportions within both internal and external features were kept constant in all the conditions. In spite of this, the holistic facial pattern changes through a combination of internal and external features in the conditions SE and SI. Moreover, in our inverted condition, both target and test faces were presented inverted (inverted-inverted), which leads to weaker inversion effects than if the procedure would have required mental rotation of the features (upright-inverted) (Rakover \& Teucher 1997). All of this indicates that facial context, defined as the configurational pattern of all presented features, may have facilitated the 
Fig. 4 Average matching errors (a) and average RTs (b) for Task 1 and a control experiment. Exposure duration in both experiments was $150 \mathrm{~ms}$ and the task was to match faces as whole. In the control experiment a pattern mask was presented and the second face resized to be $17 \%$ smaller than the target face. No differences in the number of the matching errors was found, although reaction times were faster in the control experiment (a)

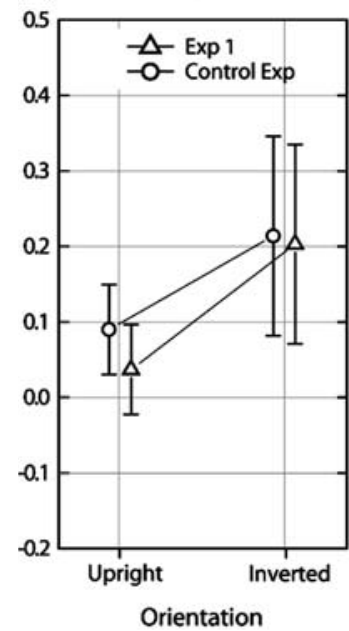

(b)

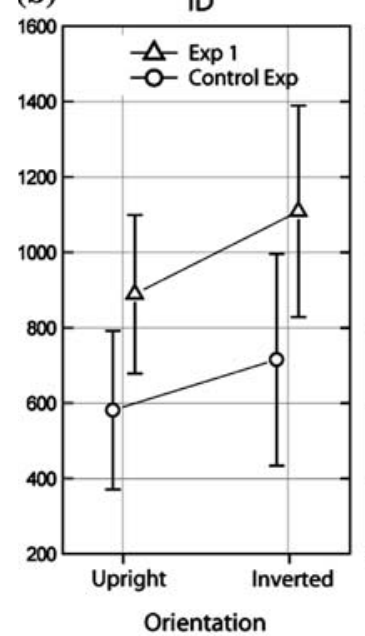

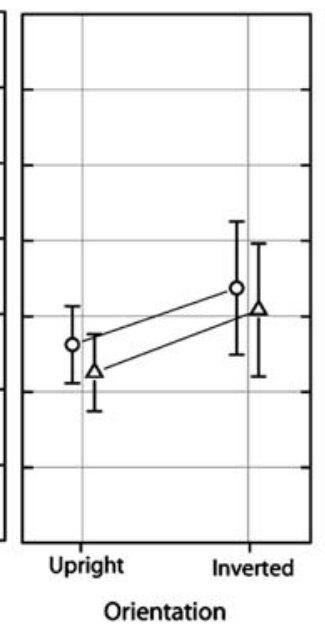

SE

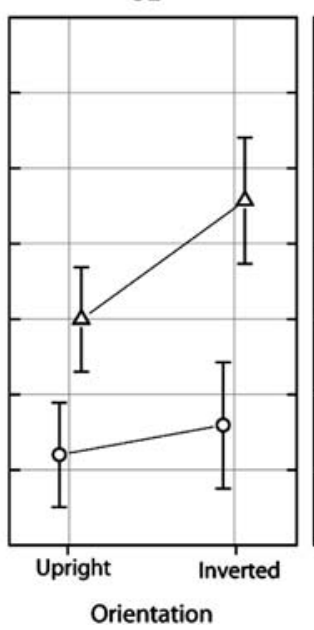

SI

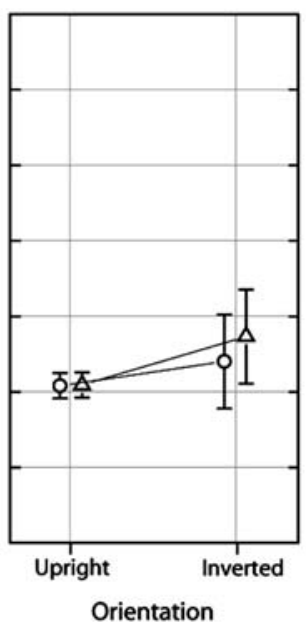

SI

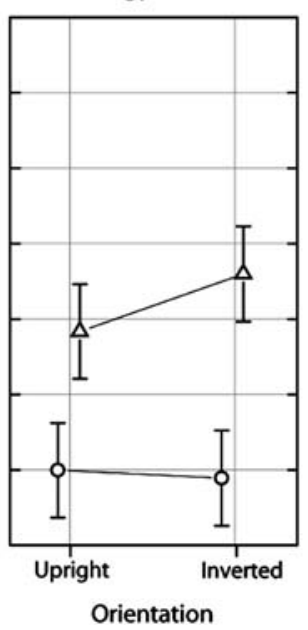

DF

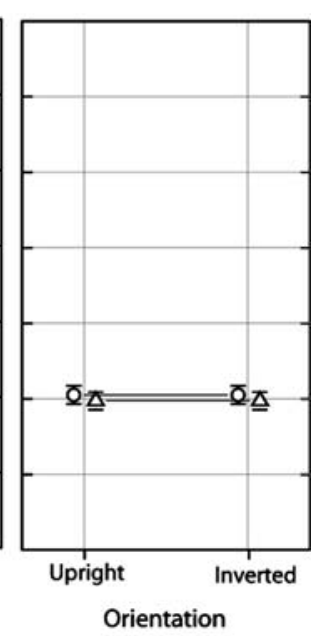

DF

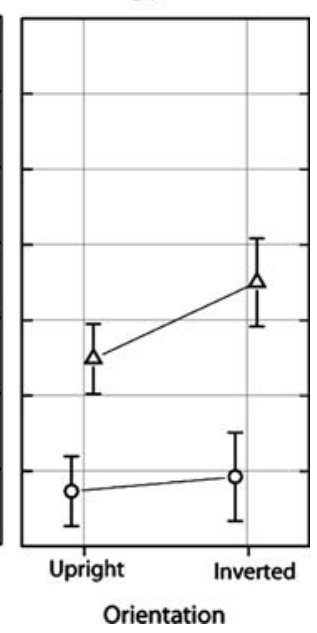

extraction of the internal and external features in inverted orientation. That would explain why inversion effect was not obtained for external and internal features in our study, although it was strong (but still weaker than for whole faces) in the studies presenting isolated features. In spite of the fact that methodological procedures could explain some aspects of the data, this assumption has a speculative character and has to be examined in further studies.

A second important aim of our study was to examine effects of exposure duration on the processing of external and internal features. We could demonstrate that within $90 \mathrm{~ms}$ exposure duration not only the whole faces, but also facial features can be extracted and matched at a level higher than chance. Although reaction times did not differ between the three tasks, the average error was the highest for matching of internal features, while there was no difference in matching of external features and whole faces. These results are in line with the previous findings suggesting more accurate matching on the basis of external rather than internal features, but failed to confirm the faster processing of external compared with internal features (De Haan \& Hay 1986; Young et al. 1985; Nachson et al. 1995). Interestingly, there was an interaction between exposure duration and orientation suggesting that processing of upright and inverted faces occurs at the different time scales. Namely, the short exposure duration of 90 and $120 \mathrm{~ms}$ resulted in a higher error rate for inverted, but not for upright stimuli. For upright faces, the maximal performance was achieved at $90 \mathrm{~ms}$ exposure duration and longer exposure durations $(120,150 \mathrm{~ms}$, self-placed) did not improve accuracy. For inverted stimuli it seems that a critical exposure duration is met at $150 \mathrm{~ms}$. Further reduction of the exposure duration to 90 or $120 \mathrm{~ms}$ decreased matching performance significantly. Prolonged exposure duration (self-paced) did not result in a significant decrease of matching errors, although there was a tendency in this direction, especially in some conditions. The reaction times in the self-paced exposure duration were significantly longer for both upright and inverted faces, but it did not result in a better performance. 
As already mentioned, the composition of a face (ID, SE, SI, DF) is supposed to have a significant impact on face matching (Nachson et al. 1995). Our data confirmed previous results by Nachson et al. (1995) that matching two different faces (condition DF) was the fastest and the most accurate. There was also a significant interaction between exposure duration and condition. Interestingly, our data showed that with longer exposure duration, the reaction time for the SE and SI conditions increased in all the three tasks; accuracy, however, did not rise with longer exposure duration. The longer reaction time for SE- and SI- trails, where the facial features have to be isolated from facial context, implicates a predominantly serial and part-based processing. In contrast, when the faces are either completely same or completely different, fast reaction times indicate a more holistic approach (ID- and DF-trails). The interaction between exposure duration and condition was modified by task, indicating that matching strategies depend on matching criteria (whole face, external or internal features). Generally, the different judgments were more accurate and faster than same judgments; i.e. in Task 1 (whole face matching), matching of identical faces (ID) was more error prone and slower than the other three conditions (SE, SI, DF). Consequently, in Task 2 (matching of external features) the highest average error score and the longest reaction time were reached with SE-trials and in Task 3 (matching of internal features) with SI-trials.

To summarize, the results in all the three tasks provide evidence for very fast face matching. Maximal performance for upright faces was achieved at $90 \mathrm{~ms}$ exposure duration, while longer exposure durations, albeit leading to longer reaction times, did not reduce the error rate. For inverted faces, performance continually decreased with exposure durations below $150 \mathrm{~ms}$. Our results are in line with previous findings suggesting that matching of unfamiliar faces is more accurate on the basis of external rather than internal features. Contrary to other studies, we could not confirm faster processing of external than internal features. However, the data implies a part-based and time-consuming process for both internal and external features if they have to be matched within facial context. Finally, this study demonstrated that manipulating exposure duration reveals some evidence about the time course in face matching.

Acknowledgments This study was supported by the Swiss National Science Foundation grant no. PMCD1-114398/1 given to Bozana Veres-Injac.

\section{References}

Barton, J. J., Keenan, J. P., \& Bass, T. (2001). Discrimination of spatial relations and features in faces: effect of inversion and viewing duration. British Journal of Psychology, 92, 527-549.
Coltheart, M. (1977). Contemporary models of the cognitive processes. I. Information input and storage. In V. Hamilton, \& M. D. Vernon (Eds.), The development of cognitive processes (pp. 1114). London: Academic Press.

Coltheart, M. (1983). Iconic memory. Philosophical transactions of the Royal Society of London Series B, Biological sciences, 302, 283-294.

De Haan, E. H. F., \& Hay, D. C. (1986). The matching of famous and unknown faces, given either the internal or the external features: a study of patients with unilateral brain lesions. In H. E. Ellis, M. A. Jeeves, F. Newcombe, \& A. W. Young (Eds.), Aspects of face processing (pp. 302-309). Dordrecht: Martinus Niijhoff.

Eger, E., Jedynak, A., Iwaki, T., \& Skrandies, W. (2003). Rapid extraction of emotional expression: evidence form evoked potential fields during brief presentation of face stimuli. Neuropsychologia, $41,808-817$.

Fuster, J. M., \& Jervey, J. P. (1981). Inferotemporal neurons distinguish and retain behaviorally relevant features of visual stimuli. Science, 212, 952-955.

Hole, G. J. (1994). Configural factors in the perception of unfamiliar faces. Peception, 23, 65-74.

Hoeger, R. (1997). Speed of processing and stimulus complexity in low-frequency and high-frequency channels. Perception, 26(8), 1039-1045.

Hubner, R. (1997). The effect of spatial frequency on global precedence and hemispheric differences. Perception and Psychophysics, 59(2), 187-201.

Jacques, C., d'Arripe, O., \& Rossion, B. (2007). The time course of the inversion effect during individual face discrimination. Journal of Vision, 7(8), 1-9.

Jeffreys, D. A. (1989). A face responsive potential recorded from the human scalp. Experimental Brain Research, 78, 193-202.

Keysers, C., Xiao, D. K., Foldiak, P., \& Perrett, D. I. (2001). The speed of sight. Journal of Cognitive Neuroscience, 13(1), 90-101.

Keysers, C., Xiao, D. K., Foldiak, P., \& Perrett, D. I. (2005). Out of sight but not out of mind: the neurophysiology of iconic memory in the superior temporal sulcus. Cognitive Neuropsychology, 22(3/4), 316-332.

Kovacs, G., Vogels, R., \& Orban, G. A. (1995). Cortical correlate of pattern backward masking. Proceedings of the National Academy of Science United States of America, 92, 5587-5591.

Love, B. C., Rouder, J. N., \& Wisniewski, E. J. (1999). A structural account of global and local processing. Cognitive Psychology, 38(2), 291-316.

Moscovitch, M., \& Moscovitch, D. A. (2000). Super face-inversion effects for isolated internal or external features, and for fractured faces. Cognitive Neuropsychology, 17, 201-219.

Martens, U., Schweinberger, S. R., Kiefer, M., \& Burton, A. M. (2006). Masked and unmasked electrophysiological repetition effects of famous faces. Brain Research, 1109(1), 146-157.

Nachson, I., Moscovitch, M., \& Umilta, C. (1995). The contribution of external and internal features to the matching of unfamiliar faces. Psychological Research, 58(1), 31-37.

Nachson, I., \& Shechory, M. (2002). Effect of inversion on the recognition of external and internal facial features. Acta Psychologica, 109(3), 227-238.

Rakover, S., \& Teucher, B. (1997). Facial inversion effects: parts and whole relationship. Perception and Psychophysics, 59, 752761.

Rolls, E. T., \& Tovee, M. J. (1994). Processing speed in the cerebral cortex and the neurophysiology of visual masking. Proceedings of the Royal Society of London B: Biological Sciences, 257, 9 15.

Schiller, P. H. (1968). Single unit analysis of backward visual masking and metacontrast in the cat lateral geniculate nucleus. Visual Research, 8, 855-866. 
Schwaninger, A., Carbon, C. C., \& Leder, H. (2003). Expert face processing specialization and constraints. In G. Schwarzer, \& H. Leder (Eds.), Development of face processing (pp. 81-97). Göttingen: Hogrefe.

Seeck, M., Michel, C. M., Mainwaring, N., Cosgrove, R., Blume, H., Ives, J., Landis, T., \& Schomer, D. L. (1997). Evidence for rapid face recognition from human scalp and intracranial electrodes. Neuroreport., 8(12), 2749-2754.
Valentine, T. (1988). Inverted faces: a review of the effect of inversion upon face recognition. British Journal of Psychology, 79, 471491.

Yin, R. K. (1970). Face recognition: a special process? Unpublished Ph.D thesis, MIT, Cambridge.

Young, A. W., Hay, D. C., McWeeny, K. H., Flude, B. M., \& Ellis, A. V. (1985). Matching familiar and unfamiliar faces on internal and external features. Perception, 14, 737-746. 